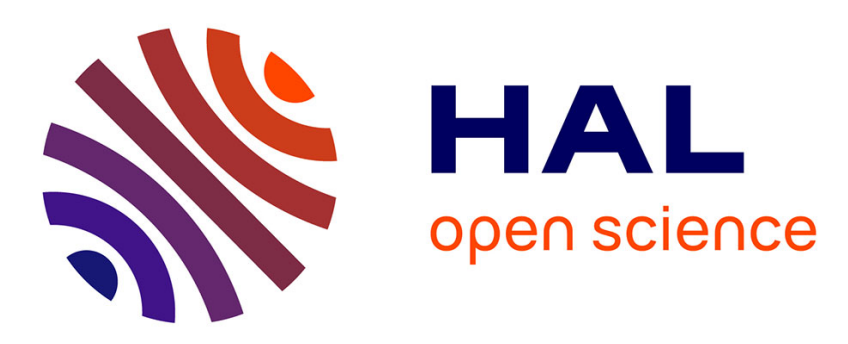

\title{
Electronic scheme for full control of organic piezoelectric MEMS resonatorss
}

Pierre-Henri Ducrot, Isabelle Dufour, Cédric Ayela, Fabrice Mathieu, Liviu

$\mathrm{Nicu}$

\section{- To cite this version:}

Pierre-Henri Ducrot, Isabelle Dufour, Cédric Ayela, Fabrice Mathieu, Liviu Nicu. Electronic scheme for full control of organic piezoelectric MEMS resonatorss. 2016 IEEE International Frequency Control Symposium (IFCS 2016), May 2016, La Nouvelle Orléans, LA, United States. 10.1109/FCS.2016.7546797 . hal-01271757

\section{HAL Id: hal-01271757 \\ https://hal.science/hal-01271757}

Submitted on 30 Aug 2016

HAL is a multi-disciplinary open access archive for the deposit and dissemination of scientific research documents, whether they are published or not. The documents may come from teaching and research institutions in France or abroad, or from public or private research centers.
L'archive ouverte pluridisciplinaire HAL, est destinée au dépôt et à la diffusion de documents scientifiques de niveau recherche, publiés ou non, émanant des établissements d'enseignement et de recherche français ou étrangers, des laboratoires publics ou privés. 


\section{Nearby electronic scheme for actuation and detection of resonance frequency and quality factor of organic piezoelectric MEMS resonators}

\author{
Pierre-Henri Ducrot, Isabelle Dufour, Cédric Ayela \\ Université de Bordeaux - CNRS - Bordeaux INP \\ Laboratoire Intégration du Matériau au Système \\ Talence, France \\ pierre-henri.ducrot@ims-bordeaux.fr
} dedicated to the measurement of the resonance frequency and the quality factor of organic piezoelectric resonators. These resonators consist of a free-standing cantilever and its nonreleased counterpart used to compensate electrical signals that are not due to mechanical behavior. The system uses a capacitive half-bridge with two AC voltages in opposite phases, associated with an IQ demodulator. The magnitudes of the two AC voltages can be set independently from one another, which enables a powerful compensation of parasitic capacitance. Thanks to this dedicated system, measurements of the resonance frequency of organic piezoelectric resonators both in air and liquid media have been performed. Several methods to determine accurately the resonance frequency have been tested and compared in terms of measurement noise.

Keywords-MEMS, resonator, electrical sensing technique, electrical compensation

\section{INTRODUCTION}

Piezoelectricity is a phenomenon that is widely used in Micro-Electro-Mechanical Systems (MEMS) because it enables, at the same time, the actuation and the detection of a motional structure with only one structural layer [1]. In order to apply an electric field in a piezoelectric material, the latter must lie between two electrodes, which leads to a capacitance in parallel with the RLC circuit that represents the mechanical behavior of the resonator. In certain cases, this capacitance is not an issue for the resonance measurement and a simple charge or voltage amplifier circuit is efficient enough [2]. But, this capacitance may be a difficulty for the electrical detection of the resonance with spectrum measurements. Other methods exist such as either a Phase-Locked Loop (PLL) system or an oscillator configuration that tracks the resonance frequency $[3,4]$. The drawback of such electronic configuration is that only the resonance frequency is determined and not the global resonance spectrum.

A common way to avoid the influence of the geometrical capacitance is based on a differential measurement between two identical structures: one is free to move while the other one is fixed. The present work introduces a compact electronic card that enables a differential measurement using a capacitive halfbridge principle and an IQ modulator. Moreover, it is possible to monitor the resonance frequency and the quality factor in real time thanks to a custom software.

\author{
Fabrice Mathieu, Liviu Nicu \\ CNRS - Université de Toulouse \\ Laboratoire d'Analyse et d'Architecture des Systèmes \\ Toulouse, France
}

\section{ELECTRONIC CARD}

\section{A. Principle}

The electronic card can generate two AC voltages in opposite phases. The magnitude of each signal can be set independently, which is an important characteristic. The measurement principle can be seen in Fig. 1. The two AC voltages supply both the free and the fixed structures. The middle point of the capacitive half-bridge is sent to a charge amplifier. The charge amplifier output signal is then sent to an IQ modulator system that gives the modulus and the phase of the resulting signal via a dedicated software connected to the card.

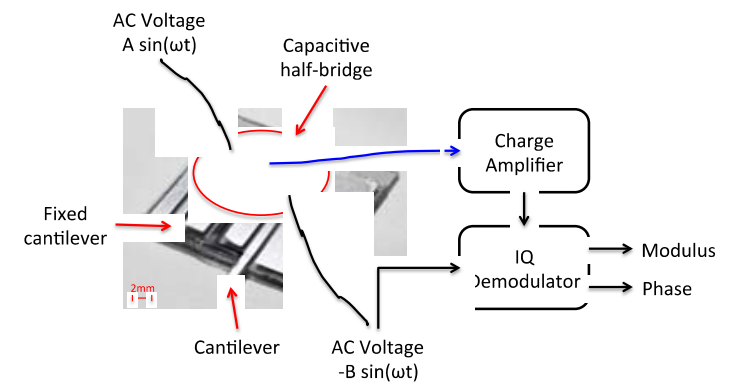

Fig. 1. Representation of the measurement principle.

\section{B. Results}

The resonators used in this work are composed of a PEN (Polyethylene naphthalate) substrate, in addition to bottom and top electrodes made of aluminum on both sides of a PVDFTrFE (polyvinylidene fluoride-trifluoroethylene) layer, which is a piezoelectric polymer [5]. In order to visualize the benefits offered by the dedicated electronic scheme, three measurements have been done in air: one without any compensation, one with compensation by the same AC voltage magnitudes for the free and the fixed structures and one with compensation with tuned voltage magnitudes (Fig. 2). It is clear that without any compensation it is really hard to observe the resonance with either the modulus or the phase spectra. The results become clearer with compensation by the same voltage magnitudes but it still does not look like a classical resonance spectrum, which means that the two structures are not exactly electrically identical. Nevertheless, with the tuned voltage magnitudes for each structure, it becomes possible to have a 
good compensation, resulting in comprehensive and usable modulus and phase signals for both quality factor and resonance frequency measurements. Using this electronic scheme and these organic piezoelectric resonators, measurements in liquids are also possible (Fig. 3). This is of particular interest for biological applications for example. The quality factor for the resonator presented in Fig. 3 is around 22 in air and 10 in water.
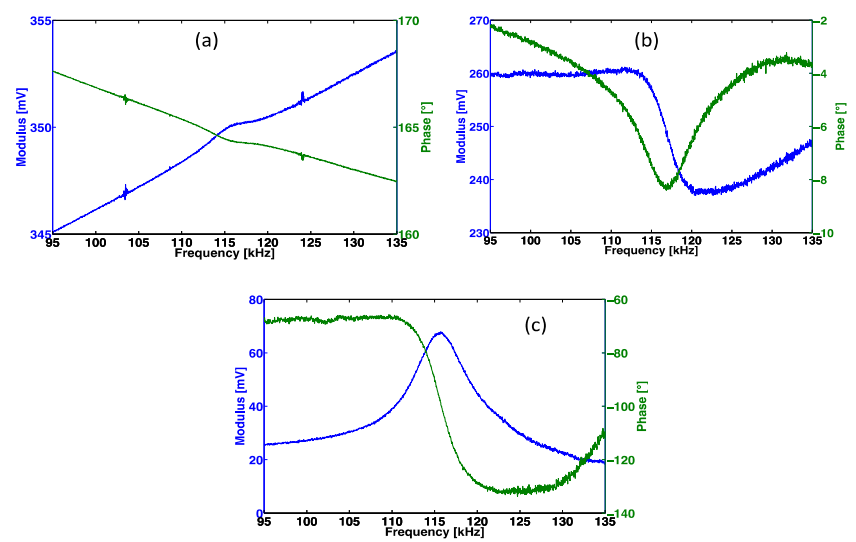

Fig. 2. Measurement of the resonance (a) without any compensation, (b) with compensation with the same AC voltage magnitudes for the free and the fixed structures and (c) with compensation with tuned voltage magnitudes.
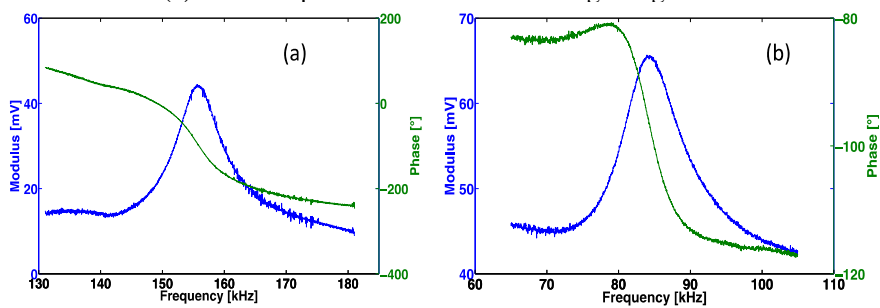

Fig. 3. Measurement of the resonance of a structure (a) in air and (b) in water.

\section{DETERMINATION OF THE RESONANCE FREQUENCY}

Once the acquisition of the resonance spectrum is done, the resonance frequency must be quantified. Several posttreatment methods have been compared in terms of measurement noise (standard deviation of the resonance frequency estimation). The first method simply gives the frequency that corresponds to the modulus maximum value. The second method consists in fitting the modulus peak with a parabola and determining the maximum value. As the parabola rapidly differs from the modulus, only data points close to the resonance must be taken into account for the fit [6]. The third method consists in fitting linearly the phase signal and determining the frequency that corresponds to a $90^{\circ}$ phase [6]. For the same reasons as previously, this fit is available only near the resonance. The last method is a treatment that determines the coupling that must be taken out in order to have a second order band-pass system [7]. Series of about fifty measurements have been recorded in air in order to calculate the mean value and the standard deviation of the resonance frequency. The results are summarized in Tab. 1. According to the results, the parabolic fit of the modulus and the linear fit of the phase give the lowest noise (260ppm which is quite good considering the low quality factor and the simplicity of the method). The developed system composed of organic piezoelectric resonators and a dedicated electronic setup enables the accurate measurement and monitoring of both the resonance frequency and the quality factor, which is of particular interest for future mass sensing assays in air and liquid media.

TABLE I. COMPARATION OF THE METHODS TO DETERMINE THE RESONANCE FREQUENCY

\begin{tabular}{|c|c|c|}
\hline Method & $\begin{array}{c}\text { Resonance } \\
\text { Frequency } \\
{[\mathrm{kHz}]}\end{array}$ & $\begin{array}{c}\text { Standard } \\
\text { Deviation } \\
{[\mathrm{Hz}]}\end{array}$ \\
\hline Parabolic fit of the modulus & 114.7 & 30 \\
\hline Linear fit of the phase & 114.3 & 34 \\
\hline Band-pass system & 114.4 & 59 \\
\hline Maximum & 114.6 & 110 \\
\hline
\end{tabular}

\section{CONCLUSION}

A compact and efficient electronic card is presented in this work. This card combines two AC voltage sources in opposite phases and an IQ modulator. This system enables the accurate compensation of the geometrical capacitance of organic piezoelectric resonators, which makes resonance frequency and quality factor measurements in liquids possible through the acquisition of the resonance spectrum. In order to determine the resonance frequency, post-treatment methods have been compared to find the best one in terms of noise level. The results show that a simple fit of the modulus by a parabola is efficient.

\section{ACKNOWLEDGMENT}

The authors acknowledge financial support by the CNRS and the University of Bordeaux (project MEMOCELL).

\section{REFERENCES}

[1] K. S. Ramadan, D. Sameoto and S. Evoy, "A review of piezoelectric polymers as functional materials for electromechanical transducers," Smart. Mater. Struct. 23, 033001, 2014.

[2] J. C. Doll and B. L. Pruitt, "Design of piezoresitive versus piezoelectric contact mode scanning probes,” J. Micromech. Microeng. 20, 095023, 2010.

[3] V. Ferrari, D. Marioli and A. Taroni, "Thick-film resonant piezo-layers as new gravimetricensors,” Meas. Sci. Technol. 8, 42-48, 1997.

[4] T. Manzaneque, V. Ruiz-Diez, J. Hernando-García, E. Wistrela, M. Kucera, U. Schmid and J. L. Sáchez-Rojas, "Piezoelectric MEMS resonator-based oscillator for density and viscosity sensing," Sens. Actuators A 220, 305-315, 2014.

[5] P.-H. Ducrot, I. Dufour and C. Ayela, "Optimization of PVDF-TrFE processing conditions for the fabrication of organic MEMS resonators," Sci. Rep. In press.

[6] M. T. Boudjiet, J. Bertrand, C. Pellet and I. Dufour, "New characterization methods for monitoring small resonant frequency variation, experimental tests in the case of hydrogen detection with uncoated silicon microcantilever-based sensors,” Sens. Actuators B 199, 269-276, 2014.

[7] A. O. Niedermayer, T. Voglhuber-Brunmaier, J. Sell and B. Jakoby, "Methods for the robust measurement of the resonance frequency and quality factor of significantly damped resonating devices,” Meas. Sci. Technol. 23, 085107, 2012. 